\title{
HD 207651: A TRIPLE SYSTEM WITH $\delta$ SCUTI AND ELLIPSOIDAL VARIATIONS BUT NO $\gamma$ DORADUS PULSATIONS
}

\author{
Gregory W. Henry, ${ }^{1}$ Francis C. Fekel, ${ }^{1,2}$ and Stephen M. Henry ${ }^{3}$ \\ Center of Excellence in Information Systems, Tennessee State University, 330 10th Avenue North, Nashville, TN 37203; \\ henry@schwab.tsuniv.edu, fekel@evans.tsuniv.edu, henrysm@galileo.tsuniv.edu \\ Received 2003 November 10; accepted 2003 November 26
}

\begin{abstract}
We examine HD 207651 as a possible example of a star exhibiting both $\gamma$ Doradus and $\delta$ Scuti type pulsations. We find photometric periods of 0.06479 and 0.06337 days with peak-to-peak amplitudes in Johnson $B$ of 21 and $13 \mathrm{mmag}$, respectively, clearly indicating $\delta$ Scuti pulsations. Additional light variation with a period of 0.73540 days and an even larger amplitude of $31 \mathrm{mmag}$ is within the range of $\gamma$ Doradus pulsation periods but results instead from the ellipticity effect. HD 207651 has a composite spectrum with a weak, narrow absorption line superposed near the center of each broad metal line. The broad-lined component is the primary of a shortperiod, single-lined binary, which has a period of 1.4708 days, twice the period of the ellipsoidal variations seen in the photometry. We determine the primary to be an A8 giant and estimate the unseen secondary of the shortperiod binary to be a mid-M dwarf. The narrow-lined star, an F7: dwarf, shows velocity variability with a period of months or perhaps years. It is thus a more distant companion to the binary, making HD 207651 a triple system. All light variations come from the A8 giant primary star. Since the 0.73540 day variation results from the ellipticity effect, HD 207651 is not an example of a star that exhibits both $\delta$ Scuti and $\gamma$ Doradus pulsations. The growing number of confirmed $\gamma$ Doradus stars that also occur within the $\delta$ Scuti instability strip but fail to show additional $\delta$ Scuti variability makes it increasingly unlikely that the two types of pulsation can coexist in the same star.
\end{abstract}

Key words: binaries: spectroscopic $-\delta$ Scuti - stars: early-type - stars: fundamental parameters stars: oscillations — stars: variables: other

On-line material: machine-readable table

\section{INTRODUCTION}

Handler (1999) found HD 207651 to have a period of 0.735 days from his analysis of Hipparcos photometry (ESA 1997) and listed it in his Table 2 as a possible $\gamma$ Doradus candidate. He also noted that it might be a $\delta$ Scuti variable. Consequently, Handler \& Shobbrook (2002) included this star in their ground-based photometric survey of confirmed and candidate $\gamma$ Doradus stars in which they searched for stars with both short-period $\delta$ Scuti and longer period $\gamma$ Doradus variability.

The $\gamma$ Doradus stars are dwarfs and subgiants that reside in a well-defined region of the H-R diagram that straddles the cool boundary of the $\delta$ Scuti instability strip. Figure 28 of Henry \& Fekel (2003), which includes all 42 of the $\gamma$ Doradus stars confirmed to date, shows that over half of them lie within the $\delta$ Scuti strip.

As discussed in the survey paper of Handler \& Shobbrook (2002), the relationship between the $\delta$ Scuti and $\gamma$ Doradus stars is not well understood. It is uncertain whether the $\delta$ Scuti and $\gamma$ Doradus pulsations are mutually exclusive or whether both types of oscillation can occur in the same star, which as Handler \& Shobbrook (2002) noted, would be extremely

\footnotetext{
${ }^{1}$ Also Senior Research Associate, Department of Physics and Astronomy, Vanderbilt University, Nashville, TN 37235.

2 Visiting Astronomer, Kitt Peak National Observatory, National Optical Astronomy Observatory, operated by the Association of Universities for Research in Astronomy, Inc., under cooperative agreement with the National Science Foundation.

${ }^{3}$ Also at Department of Science and Mathematics, Trevecca Nazarene University, Nashville, TN 37210.
}

interesting for asteroseismology. Also, for stars that lie within both instability strips, it is not known what determines the type of pulsation that occurs in a particular case or if observable pulsation will be present at all.

In their examination of six confirmed $\gamma$ Doradus variables lying within the intersection of the $\delta$ Scuti and $\gamma$ Doradus instability strips, Handler \& Shobbrook (2002) found only one star, HD 209295, that showed both types of variability. However, Handler et al. (2002) argued that the $\gamma$ Doradus-type pulsations in this star are probably excited by the presence of a degenerate companion in an elliptical orbit, making this star anomalous. The survey of Handler \& Shobbrook (2002) found both short-term $\delta$ Scuti type variability with a timescale of $1.5-2 \mathrm{hr}$ and additional longer term variability in HD 207651, but they could not determine the cause of the long-term variability. For three additional candidates they found that the longer term variability results from ellipsoidal variations in binary systems. For HD 207651, they noted a 1.2 mag discrepancy in the absolute magnitudes determined from the star's Hipparcos parallax and from its Strömgren photometry and suggested that this star might also be a binary. As a result of their failure to find $\delta$ Scuti and $\gamma$ Doradus pulsations in the same star, except for the unusual case of HD 209295, Handler \& Shobbrook (2002) concluded that $\gamma$ Doradus stars within the $\delta$ Scuti instability strip are less likely to be $\delta$ Scuti pulsators than non $-\gamma$ Doradus stars located in the same region of the H-R diagram. However, they pointed out the need to study additional stars in the overlap region.

Fekel et al. (2003) included HD 207651 in their spectroscopic survey of over $30 \gamma$ Doradus candidates listed by Handler (1999). They obtained a single spectrum of it that 
shows composite line profiles consisting of a narrow component situated near the center of each broad-lined component. They classified the broad component as A9 and noted that the Hipparcos parallax indicates that the star is a giant. The radial velocities of the broad- and narrow-lined stars were similar to each other but differed by about $20 \mathrm{~km} \mathrm{~s}^{-1}$ from the mean velocity of Fehrenbach et al. (1997), obtained from objectiveprism spectra, suggesting that the system is indeed a shortperiod binary. Fekel et al. (2003) also reported that two nights of additional photometry by Handler (2002, private communication) suggested that the long-period brightness variations are the result of duplicity, based on a lack of detectable color variations.

Because of the composite nature of its spectrum and the presence of both short- and long-period photometric variations, we obtained additional photometric and spectroscopic observations to determine the physical properties of HD 207651, measure the periods of photometric variability, and verify their cause or causes. Our new photometric and spectroscopic observations are described in $\S \S 2$ and 3, respectively, and our results are discussed in $\S 4$.

\section{PHOTOMETRY}

\subsection{Observations}

The photometric observations were acquired between 2001 September and 2002 October with the T3 $0.4 \mathrm{~m}$ automatic photoelectric telescope (APT) at Fairborn Observatory. The $0.4 \mathrm{~m}$ APT uses a temperature-stabilized EMI 9924B photomultiplier tube to acquire data successively through Johnson $B$ and $V$ filters. The measurements of variability in HD 207651 were made in our usual group observation sequence: $K, S, C$, $V, C, V, C, V, C, S, K$, in which $K$ is a check star, $C$ is the comparison star, $V$ is the variable star, and $S$ is a sky reading. The comparison star was HD $208897(V=6.50, B-V=1.01$, $\mathrm{K} 0)$; the check star was HD $207719(V=7.24, B-V=1.10$, K0 III). Between 2001 September and 2002 July, up to five group observations were acquired per night at intervals of about 2 hr. Four consecutive nights in 2002 October were devoted to monitoring the group for several hours each night and resulted in 50-60 group observations per night. In all, over 400 complete groups observations were obtained.

Three variable minus comparison $(V-C)$ and two check minus comparison $(K-C)$ differential magnitudes were formed from each sequence and averaged together to create group means. Group mean differential magnitudes with internal standard deviations greater than 0.01 mag were rejected to filter the observations taken under nonphotometric conditions. The surviving group means were corrected for differential extinction with nightly extinction coefficients, transformed to the Johnson system with yearly mean transformation coefficients, and treated as single observations thereafter. The external precision of our differential magnitudes, defined as the standard deviation of a single differential magnitude from the seasonal mean of the differential magnitudes and determined from observations of pairs of constant stars, is $\sim 0.005 \mathrm{mag}$. Further information on the operation of the APT and the analysis of the data can be found in Henry (1995a, 1995b). The individual photometric observations are given in Table 1. The entire set of $B$ observations are plotted versus Julian Date in the top panel of Figure 1, while the $B$ data from the four monitoring nights are shown separately plotted to the same $y$-axis scale in the remaining panels.

\subsection{Period Analysis}

We applied the method of Vanicek (1971), as described in Henry et al. (2001), to search for periodicities in the photometric data. We first analyzed the check star minus comparison star $(K-C)$ data in $B$ and $V$ over the frequency range $0.01-$ 30.0 day $^{-1}$, which corresponds to the period range $0.033-$ 100 days, and found no evidence for periodicity in either the check or comparison stars. The standard deviation of the $K-C$ observations is $0.005 \mathrm{mag}$ in both the $B$ and the $V$ photometric bands, so the check and comparison stars are constant to within the precision of our measurements. We then analyzed the variable minus comparison $(V-C)$ differential magnitudes in $B$ and $V$ over the same frequency range. The resulting power spectra for the $B$ data are shown in Figure 2, and the complete photometric results are summarized in Table 2. The top panel of Figure 2 shows the power spectrum resulting from the full $B$ data set consisting of 421 observations; clear evidence of frequencies in the range 10-20 day ${ }^{-1}$ typical of $\delta$ Scuti variability is seen as well as even stronger variability of lower frequency typical of $\gamma$ Doradus variability. The highest peak in the top panel, marked with an arrow, corresponds to a frequency of $1.3598 \mathrm{day}^{-1}$ or a period of 0.73540 days. The same period is found in our analysis of the full $V$ data set containing 411 observations (Table 2). Doubling this period gives 1.4708 days, which is consistent with the radial velocity period (see $\S 3.3$ below). Therefore, we interpret this component of HD 207651's variability as being due to the ellipsoidal shape of the broad-lined Aa component in this triple system. Further evidence of this interpretation comes from the comparison of the peak-to-peak amplitudes of the $B$ and $V$ data sets at this period: $30.7 \pm 1.7$ and $29.4 \pm 1.5 \mathrm{mmag}$, respectively (Table 2 ). Thus, the $B / V$ amplitude ratio is 1.04 , very close to unity and indicative of a geometric rather than a pulsational origin of the variability. Finally, confirmation that the ellipticity effect arises in the Aa component comes from comparison of the time of photometric

TABLE 1

Photometric Observations of HD 207651

\begin{tabular}{|c|c|c|c|c|}
\hline $\begin{array}{c}\text { Date } \\
(\mathrm{HJD}-2,400,000)\end{array}$ & $\begin{array}{l}\operatorname{Var} B \\
(\mathrm{mag})\end{array}$ & $\begin{array}{l}\text { Var } V \\
\text { (mag) }\end{array}$ & $\begin{array}{l}\text { Chk } B \\
\text { (mag) }\end{array}$ & $\begin{array}{l}\text { Chk } V \\
\text { (mag) }\end{array}$ \\
\hline 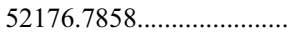 & -0.054 & 0.718 & 0.833 & 0.738 \\
\hline 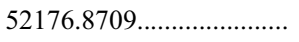 & -0.092 & 0.675 & 0.834 & 0.741 \\
\hline 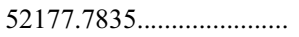 & -0.072 & 0.691 & 0.824 & 0.734 \\
\hline $52177.8677 \ldots \ldots \ldots \ldots \ldots \ldots \ldots$ & -0.051 & 0.722 & 0.817 & 0.730 \\
\hline 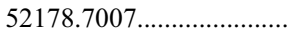 & -0.041 & 0.728 & 0.825 & 0.737 \\
\hline
\end{tabular}

Note.-Table 1 is presented in its entirety in the electronic edition of the Astronomical Journal. A portion is shown here for guidance regarding its form and content. 

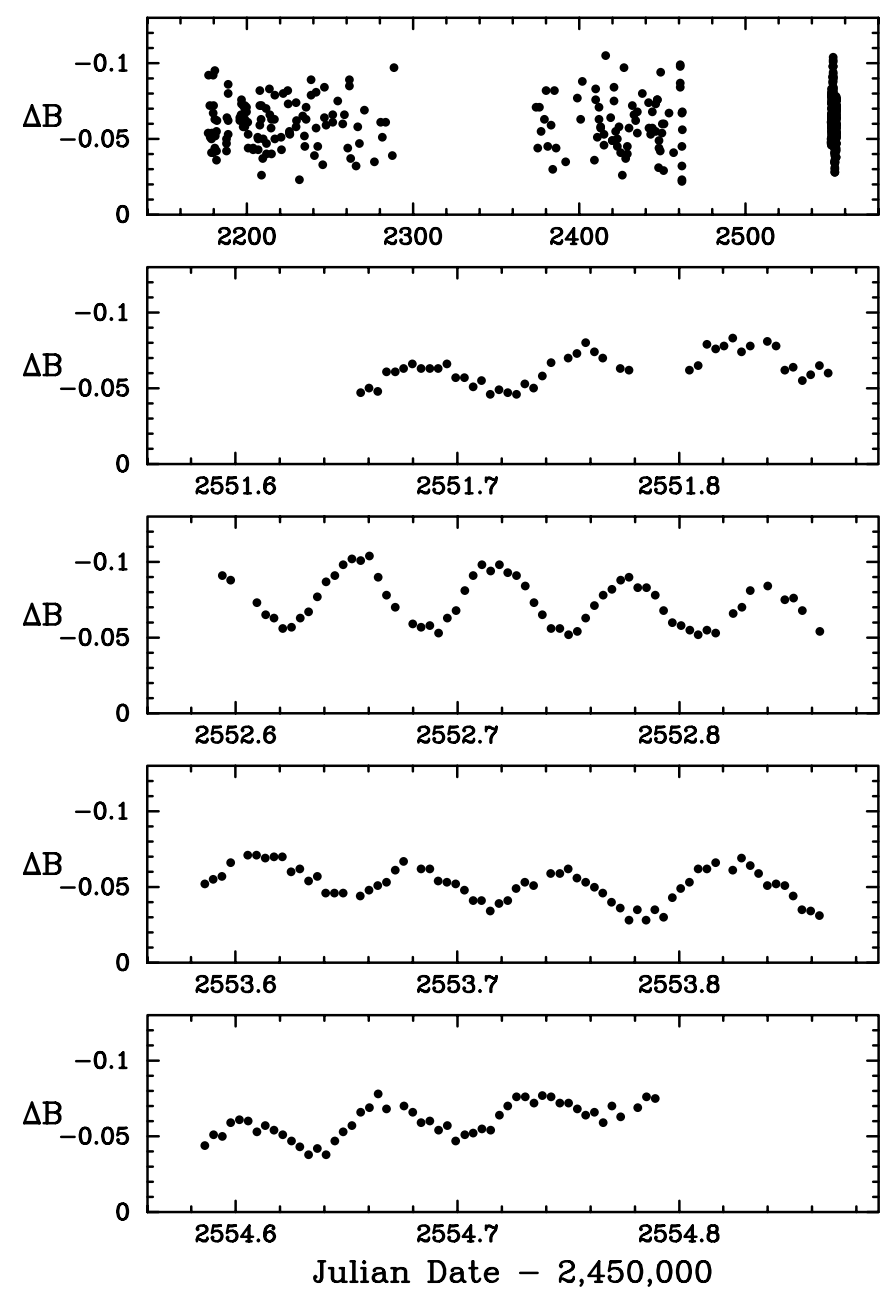

FIG. 1.-Top panel shows the complete set of $B$ photometric observations of HD 207651 acquired between 2001 September and 2002 October. The $B$ observations from the four individual monitoring nights in 2002 October are replotted separately in the remaining panels.

minimum and the time of radial velocity maximum of the Aa component ( $\$ 3.3)$.

When we fixed the 1.3598 day $^{-1}$ frequency and attempted to find the higher frequencies from the full $B$ and $V$ data sets, strong 1 cycle day ${ }^{-1}$ aliasing prevented us from unambiguously identifying the higher frequencies. To minimize these difficulties in our single-site data, we decided to use the full $B$ and $V$ data sets only to fix the frequency associated with the ellipticity effect, which could be determined unambiguously and with high accuracy, and then determine the higher $\delta$ Scuti frequencies from the four 2002 October monitoring nights alone. The second panel (from the top) of Figure 2 shows the power spectrum resulting from the four monitoring nights in $B$; the arrow marks the 1.3598 day $^{-1}$ frequency determined from the full $B$ data set. The third panel shows the power spectrum for the four nights with the 1.3598 day $^{-1}$ frequency fixed; the strongest frequency here is $15.43 \mathrm{day}^{-1}$, corresponding to a period of 0.06479 days. Fixing this frequency reveals another strong frequency at $15.78 \mathrm{day}^{-1}$, corresponding to a period of 0.06337 days (fourth panel). These two high frequencies differ by only $0.35 \mathrm{day}^{-1}$, so they are only marginally resolved in our short monitoring data set according to the criterion of Loumos \& Deeming (1978). Consequently, the uncertainties of these frequencies are likely

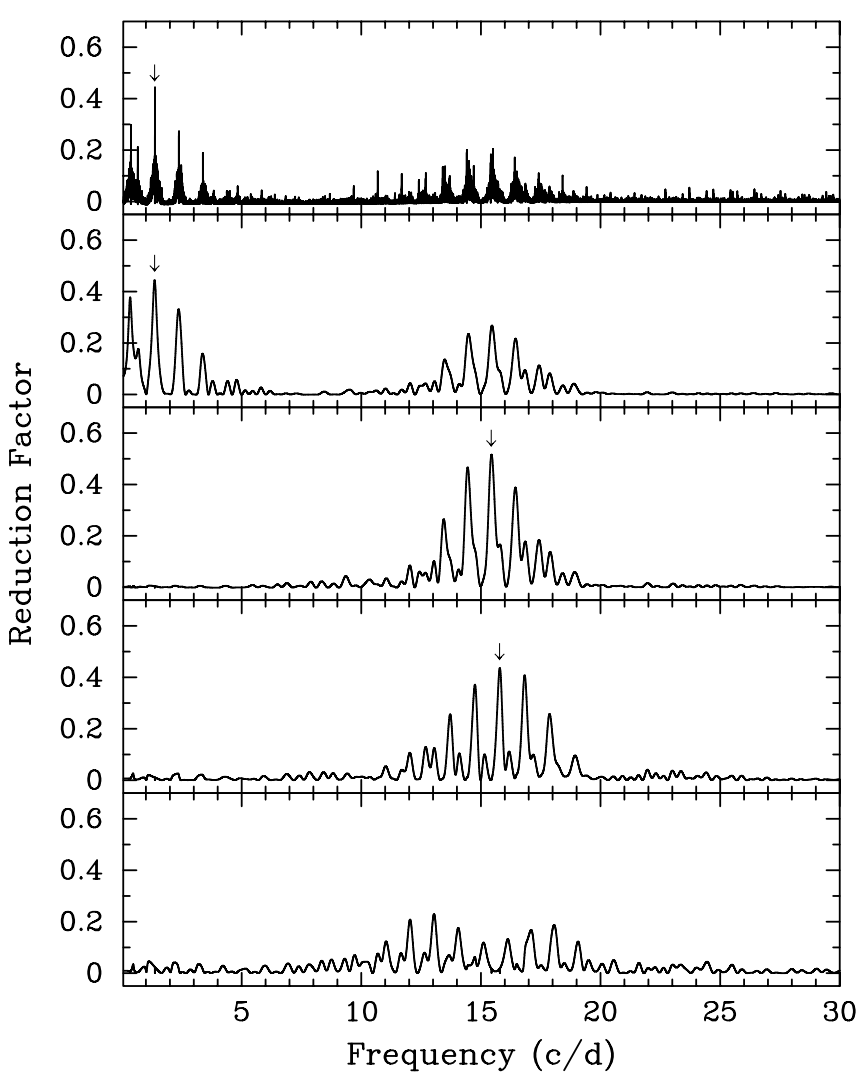

FIG. 2.-Power spectra of the HD $207651 B$ data, showing the results of progressively fixing the three detected frequencies. The top panel results from the analysis of the entire $B$ data set; the remaining panels are the power spectra of the 2002 October monitoring data only. The three detected frequencies are $1.3598,15.43$, and 15.78 day $^{-1}$.

to be significantly larger than our formal uncertainties given in Table 2. Finally, the bottom panel of Figure 2 shows the power spectrum with all three frequencies fixed. There is evidence of additional variability in the $10-20$ day $^{-1}$ frequency range, but as a consequence of 1 cycle day $^{-1}$ aliasing, we cannot identify additional frequencies with any confidence. The standard deviation of the $B$ monitoring data is 0.015 mag before any of the three frequencies are removed; it is reduced to only $0.006 \mathrm{mag}$ with the removal of the three frequencies. Therefore, given our measurement precision of $\sim 0.005$ mag, most of the variability in HD 207651 is accounted for by these three frequencies.

We obtained essentially identical results in our analysis of the $V$ data (Table 2). Our $\delta$ Scuti periods are consistent with the $1.5-2 \mathrm{hr}$ variability timescale from Handler \& Shobbrook (2002). The peak-to-peak amplitudes reported in column (6) are determined for each frequency without prewhitening for the other frequencies. Times of minimum light for each frequency are given in column (7); the times of minimum in the two passbands agree within their uncertainties, so there are no detectable phase shifts in our two-color photometry. The $B$ amplitudes average 1.21 times larger than the $V$ amplitudes for the two $\delta$ Scuti frequencies, confirming that the high frequencies arise from pulsation.

The four nights of monitoring data in $B$ are phased with each of the three frequencies and times of minimum from Table 2 and plotted in Figure 3. In each panel, the data have been prewhitened to remove the other two frequencies to improve the visibility of the brightness variations. The light 
TABLE 2

Рнотоmetric Results on HD 207651

\begin{tabular}{|c|c|c|c|c|c|c|}
\hline $\begin{array}{l}\text { Photometric Band } \\
\text { (1) }\end{array}$ & $\begin{array}{c}\text { Date Range } \\
\text { (HJD - } 2,400,000) \\
(2)\end{array}$ & $\begin{array}{l}N_{\text {obs }} \\
\text { (3) }\end{array}$ & $\begin{array}{l}\text { Frequency } \\
\left(\text { day }^{-1}\right) \\
(4)\end{array}$ & $\begin{array}{l}\text { Period } \\
\text { (days) } \\
(5)\end{array}$ & $\begin{array}{l}\text { Peak-to-Peak Amplitude } \\
\text { (mmag) } \\
(6)\end{array}$ & $\begin{array}{c}T_{\min } \\
(\mathrm{HJD}-2,450,000) \\
(7)\end{array}$ \\
\hline \multirow[t]{3}{*}{$B$} & $52,176.7858-52,554.7899$ & 421 & $1.3598 \pm 0.0001$ & $0.73540 \pm 0.00005$ & $30.7 \pm 1.7$ & $52,553.044 \pm 0.006$ \\
\hline & $52,551.6550-52,554.7899$ & 227 & $15.43 \pm 0.01^{\mathrm{a}}$ & $0.06479 \pm 0.00005^{\mathrm{a}}$ & $21.4 \pm 2.4$ & $52,553.010 \pm 0.001$ \\
\hline & & & $15.78 \pm 0.01^{\mathrm{a}}$ & $0.06337 \pm 0.00004^{\mathrm{a}}$ & $12.9 \pm 2.7$ & $52,553.001 \pm 0.002$ \\
\hline \multirow[t]{3}{*}{$V$} & $52,176.7858-52,554.7899$ & 411 & $1.3599 \pm 0.0001$ & $0.73535 \pm 0.00005$ & $29.4 \pm 1.5$ & $52,553.031 \pm 0.006$ \\
\hline & $52,551.6550-52,554.7899$ & 224 & $15.45 \pm 0.01^{\mathrm{a}}$ & $0.06473 \pm 0.00005^{\mathrm{a}}$ & $17.1 \pm 2.2$ & $52,553.009 \pm 0.001$ \\
\hline & & & $15.79 \pm 0.01^{\mathrm{a}}$ & $0.06333 \pm 0.00004^{\mathrm{a}}$ & $11.0 \pm 2.4$ & $52,553.001 \pm 0.002$ \\
\hline
\end{tabular}

${ }^{a}$ The two higher frequencies are marginally resolved in the short data set, so the errors are likely to be larger than our formal errors given here.

curves at all three frequencies are approximately sinusoidal, but some additional variability or scatter about these sinusoids is present in all three panels, suggesting that additional, weak frequency components may be present as noted above.

\section{SPECTROSCOPY}

\subsection{Observations}

From 2000 July to 2003 October we obtained nine highresolution spectrograms with the Kitt Peak National Observatory (KPNO) coudé feed telescope, coudé spectrograph, and a TI CCD detector. Eight are centered in the red at $6430 \AA$, cover a wavelength range of about $80 \AA$, and have a resolution of $0.21 \AA$. A single spectrogram was taken at a blue wavelength of $4500 \AA$ and has the same wavelength range and resolution as our other eight observations. The spectra have typical signal-to-noise ratios of 150-200.

As shown by Fekel et al. (2003) and seen in Figure 4, the spectrum of HD 207651 has two sets of features, a narrowlined component and a broad-lined component. Radial velocities of both components were determined with the IRAF

${ }^{4}$ IRAF is distributed by the National Optical Astronomy Observatory.

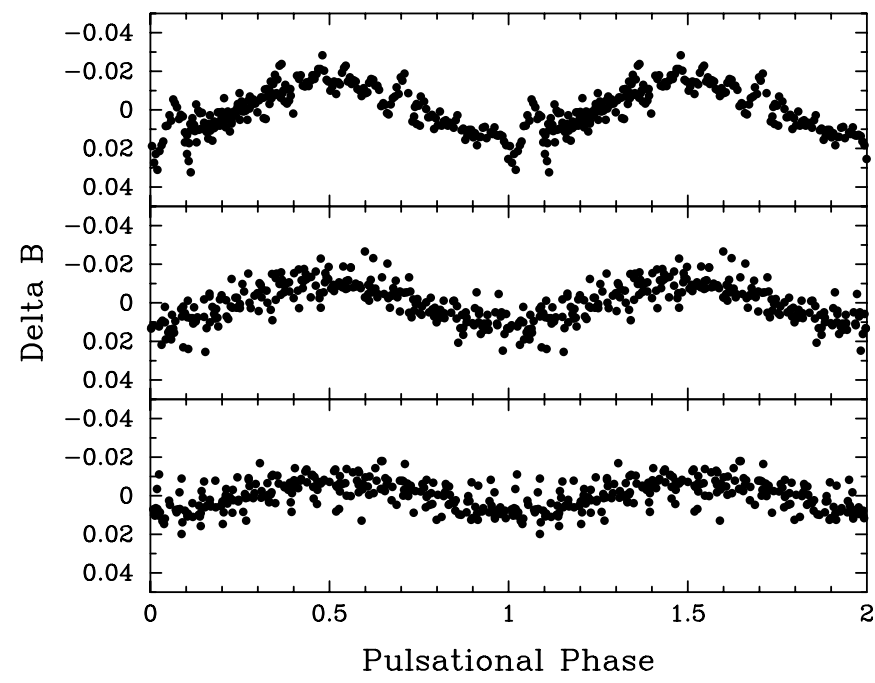

FIG. 3.-HD 207651 Johnson $B$ photometric monitoring data from 2002 October, phased with the three frequencies and times of minimum from Table 2. Top to bottom: Frequencies are 1.3598, 15.43, and $15.78 \mathrm{day}^{-1}$. For each panel, the data set has been prewhitened to remove the other two known frequencies. The variability in the top panel is due to the ellipsoidal shape of the Aa component of the HD 207651 triple system. The other two frequencies arise from $\delta$ Scuti pulsations in the same component. cross-correlation program FXCOR (Fitzpatrick 1993). The IAU radial velocity standards $\iota$ Piscium and HR 7560 were used as reference stars. Their velocities of 5.6 and $0.0 \mathrm{~km} \mathrm{~s}^{-1}$, respectively, were adopted from Scarfe, Batten, \& Fletcher (1990). The star 68 Tauri was used as the radial-velocity reference star to cross-correlate the blue spectrum. Its velocity of $39.0 \mathrm{~km} \mathrm{~s}^{-1}$ was adopted from Fekel (1999). For the narrow-lined star, called component B, three or four lines typically were measured, while for the broad-lined star, called component Aa, only one or two of the least blended lines were measured. Because of much greater line broadening, the superposed narrow features of $\mathrm{B}$, and line asymmetries caused by pulsation, the radial velocities of Aa are more uncertain than those of $\mathrm{B}$. Based on previous experience, we estimate uncertainties of $2-3 \mathrm{~km} \mathrm{~s}^{-1}$ for the velocities of Aa and $0.5 \mathrm{~km} \mathrm{~s}^{-1}$ for B. For component B, Table 3 lists the Heliocentric Julian Dates and radial velocities of the nine observations. The same information is given in Table 4 for component Aa, the broad-lined star. Fekel et al. (2003)

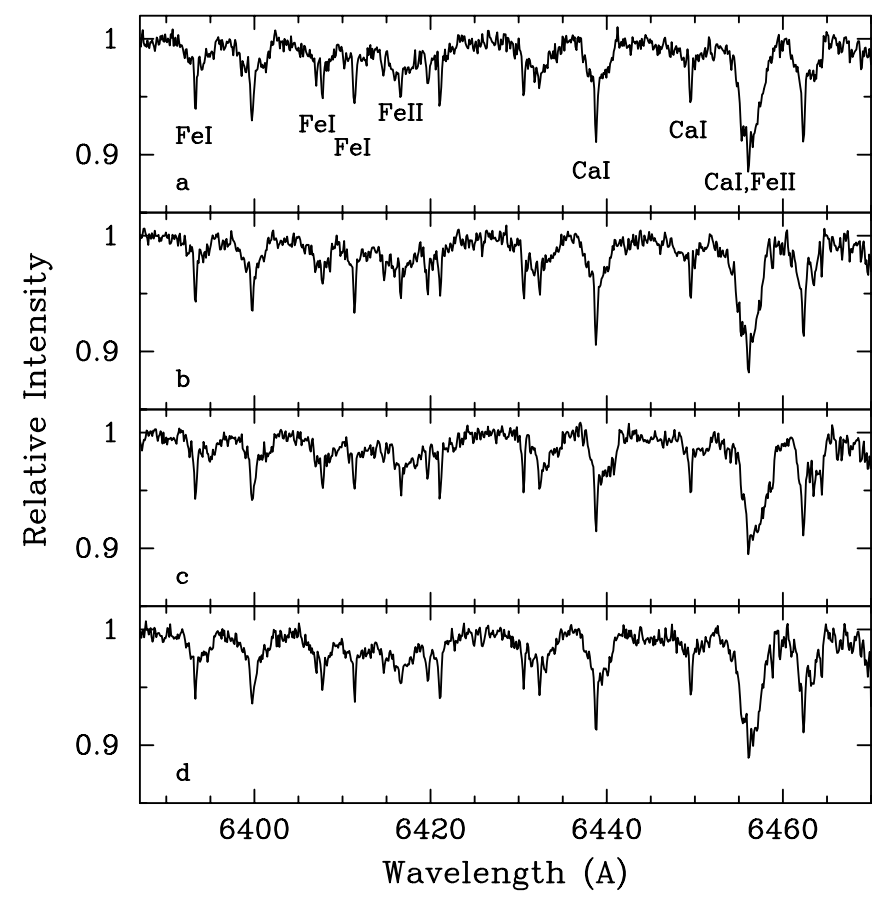

FIG. 4.-Red-wavelength spectra taken on four consecutive nights showing the composite profiles of the metal lines. The Julian Dates of the observations are (a) 2,452,539, (b) 2,452,540, (c) 2,452,541, and (d) 2,452,542. Note the shift of the broad features relative to the narrow lines. The element and ionization stage of some of the lines are indicated. 
TABLE 3

Radial Velocities of HD 207651 B

\begin{tabular}{|c|c|}
\hline $\begin{array}{c}\text { Date } \\
\text { (HJD }-2,400,0000)\end{array}$ & $\begin{array}{l}\text { Velocity } \\
\left(\mathrm{km} \mathrm{s}^{-1}\right)\end{array}$ \\
\hline $51,737.902 \ldots$ & -20.7 \\
\hline 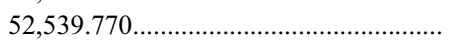 & -22.7 \\
\hline $52,540.756 \ldots \ldots \ldots \ldots$ & -23.1 \\
\hline 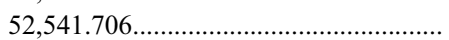 & -23.4 \\
\hline 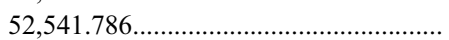 & $-23.1^{\mathrm{a}}$ \\
\hline $52,542.705 \ldots \ldots$ & -24.2 \\
\hline 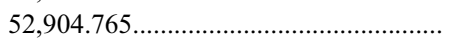 & 6.3 \\
\hline 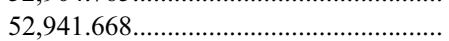 & 12.1 \\
\hline $52,942.690 \ldots \ldots \ldots$ & 12.9 \\
\hline
\end{tabular}

a The 4500 Å region.

previously listed the radial velocities of the single spectrum that was obtained in 2000 July.

\subsection{The Narrow-lined Star, Component B}

Fekel et al. (2003) found the projected rotational velocity of the narrow-lined star to be $6 \mathrm{~km} \mathrm{~s}^{-1}$. The five radial velocities of that component, obtained on four consecutive nights in 2002 September, have a mean value of $-23.3 \pm 0.3 \mathrm{~km} \mathrm{~s}^{-1}$. Since its velocity is constant during that interval, the narrow-lined component is not the secondary of the short-period binary but rather a third star in the system, component $\mathrm{B}$. Its mean velocity for 2002 September differs by $18 \mathrm{~km} \mathrm{~s}^{-1}$ from the centerof-mass velocity of the short-period binary. The two velocities of component B obtained 13 months later in 2003 October show the radial velocity of $B$ has increased by nearly $36 \mathrm{~km} \mathrm{~s}^{-1}$ (Fig. 5). With velocities obtained at only four epochs the long period cannot be reliably determined. However, several possibilities suggest themselves. One or perhaps two cycles may have occurred during the 800 day time interval between our first and second observations. Alternatively, the period may be greater than 1200 days, the full time span of our velocities. With the Hipparcos parallax (ESA 1997) and masses estimated in $\S 4$, Kepler's third law produces a semimajor axis of 0.005 for a period of 400 days and $0 " 012$ for a period of 1500 days. However, the Hipparcos observations (ESA 1997) provide no evidence that HD 207651 is a binary.

\subsection{Orbit of the Broad-lined Star, Component Aa}

Fekel et al. (2003) determined the projected rotational velocity of component Aa to be $95 \mathrm{~km} \mathrm{~s}^{-1}$. Our nine radial

TABLE 4

Radial Velocities of HD 207651 Aa

\begin{tabular}{ccccc}
\hline \hline $\begin{array}{c}\text { Date } \\
(\mathrm{HJD}-2,400,000)\end{array}$ & Phase & $\begin{array}{c}\text { Velocity } \\
\left(\mathrm{km} \mathrm{s}^{-1}\right)\end{array}$ & $\begin{array}{c}O-C \\
\left(\mathrm{~km} \mathrm{~s}^{-1}\right)\end{array}$ & Weight \\
\hline $51,737.902 \ldots \ldots \ldots \ldots .$. & 0.513 & -24.4 & -1.3 & 1.0 \\
$52,539.770 \ldots \ldots \ldots \ldots \ldots .$. & 0.705 & -11.2 & -1.1 & 1.0 \\
$52,540.756 \ldots \ldots \ldots \ldots .$. & 0.375 & -16.8 & 1.1 & 1.0 \\
$52,541.706 \ldots \ldots \ldots \ldots .$. & 0.021 & 13.5 & 0.4 & 1.0 \\
$52,541.786 \ldots \ldots \ldots \ldots .$. & 0.076 & $10.5^{\mathrm{a}}$ & -0.8 & 1.0 \\
$52,542.705 \ldots \ldots \ldots \ldots \ldots$. & 0.700 & -8.8 & 1.8 & 1.0 \\
$52,904.765 \ldots \ldots \ldots \ldots .$. & 0.866 & -0.1 & -7.3 & 0.0 \\
$52,941.668 \ldots \ldots \ldots \ldots .$. & 0.956 & -5.4 & -18.0 & 0.0 \\
$52,942.690 \ldots \ldots \ldots \ldots \ldots$. & 0.651 & -32.7 & -17.1 & 0.0 \\
\hline
\end{tabular}

a The $4500 \AA$ Å region. velocities of this broad-lined component have a range of over $55 \mathrm{~km} \mathrm{~s}^{-1}$ (Table 4), but a portion of this variation results from motion in the long-period orbit. As a result, we have used only the first six radial velocities to determine the short-period orbit; the last three velocities have been given zero weight. The velocity changes of Aa relative to B can be seen in our red-wavelength spectra (Fig. 4), taken on four consecutive nights. Five additional velocities of Fehrenbach et al. (1997) are not sufficiently precise to be useful in a period search. Thus, with only six radial velocities, five of which were obtained on four consecutive days, the prospect of a welldetermined spectroscopic period is not good. Nevertheless, we searched for the orbital period by fitting a sine curve to the velocities for trial periods between 0.1 and 10 days with a step size of 0.0001 days. For each period the sum of the squared residuals is computed, and the period with the smallest value of that sum is identified as the preliminary value of the orbital period. In this case a period of 1.4495 days has the smallest sum of the squared residuals. However, because of the small number of velocities there are other possible periods that are nearly as good.

Fortunately, our photometry enables us to identify a welldetermined orbital period from the $0.73540 \pm 0.00005$ day period caused by the ellipticity effect (see $\S 2.2$ ). When multiplied by a factor of 2 , this produces an orbital period of 1.4708 days, a value similar to the spectroscopic period listed above. This photometrically determined period has been adopted in our orbital solution. Because the period is so short, the orbit is expected to be circularized (Matthews \& Mathieu 1992), and so we have set $e=0$. The resulting orbital elements are given in Table 5. Since a time of periastron passage is undefined in a circular orbit, as recommended by Batten, Fletcher, \& MacCarthy (1989), $T_{0}$, a time of maximum radial velocity, is listed instead. This value should differ by onequarter phase from the time of minimum, $T_{\min }$, listed in Table 2 for the ellipticity effect. When one-fourth of the orbital period is added to $T_{\min }$ and compared with $T_{0}$ from the orbit determination, the difference is 0.03 days. This is only a factor of 2 larger than the 0.014 day formal uncertainty of $T_{0}$ (Table 5), confirming the ellipticity effect interpretation.

The orbital phases and velocity residuals of the orbital solution are included in Table 4. The standard error of an observation of unit weight is $1.6 \mathrm{~km} \mathrm{~s}^{-1}$, not unreasonable given the difficulty in measuring the weak, broad features. The six observed velocities that were given unit weight and the computed velocity curve are compared in Figure 6, where zero phase is a time of maximum velocity.

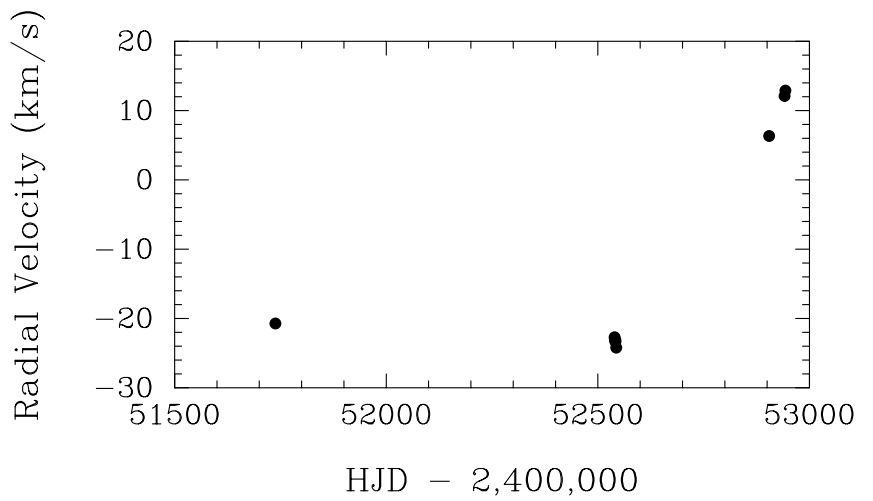

FIG. 5.-Radial velocities of component B vs. Heliocentric Julian Date 
TABLE 5

Orbital Elements of HD 207651 Aa

\begin{tabular}{|c|c|}
\hline Parameter & Value \\
\hline$P \ldots \ldots$ & 1.4708 days (adopted) \\
\hline$T_{0} \ldots \ldots \ldots \ldots \ldots$ & $2,452,553.441 \pm 0.014 \mathrm{HJD}$ \\
\hline (n) & $-4.95 \pm 0.67 \mathrm{~km} \mathrm{~s}^{-1}$ \\
\hline 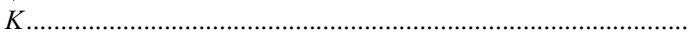 & $18.24 \pm 0.90 \mathrm{~km} \mathrm{~s}^{-1}$ \\
\hline e & 0.0 (adopted) \\
\hline 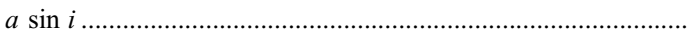 & $0.369 \pm 0.018 \times 10^{6} \mathrm{~km}$ \\
\hline$f(m)$ & $0.00093 \pm 0.00014 M_{\odot}$ \\
\hline Standard error of an observation of unit weight....................... & $1.6 \mathrm{~km} \mathrm{~s}^{-1}$ \\
\hline
\end{tabular}

\subsection{Spectral Classification}

HD 207651 is one of seven composite-spectrum stars that Fekel et al. (2003) suggested might be shell stars. Henry \& Fekel (2003) concluded that the other six are binaries, and we have found that the composite nature of the spectrum of HD 207651 also results from duplicity. Fekel et al. (2003) classified the broad-lined component as A9, and the Hipparcos parallax indicates that it is a giant. Fekel et al. (2003) suggested the spectrum of the narrow-lined component has a similar or somewhat later spectral type. With new blue- and redwavelength spectra of HD 207651 and several additional spectra of various reference stars, we reexamined the spectral classifications of the components with the same method discussed by Fekel et al. (2003). We conclude that the spectral class of the broad-lined star is A8, quite similar to the result of Fekel et al. (2003), and that of the narrow-lined star is F7:. The continuum magnitude difference at $6430 \AA$ is $2.0 \mathrm{mag}$. This is a minimum value of the actual magnitude difference since the fainter star has a later spectral class.

\section{DISCUSSION}

The Hipparcos parallax for HD 207651 is $0.00296 \pm$ 0.00077 (ESA 1997). Adopting the minimum magnitude difference of 2.0 mag (above) and assuming no interstellar extinction, we find that the broad-lined primary component Aa, which we have classified as an A8 star, has $M_{v}=-0.3 \pm$ $0.5 \mathrm{mag}$. This result is incompatible with the mid to late $\mathrm{F}$ spectral classification of component $\mathrm{B}$, which is likely a dwarf given the improbability of finding both the Aa and B components within the Hertzsprung gap. Thus, the B component should have $M_{v} \sim 3.9 \mathrm{mag}$ (Gray 1992). If it were indeed 4 mag fainter than Aa, it would not be seen in our spectra. This suggests that the Hipparcos parallax is too small. Perhaps its measurement was systematically affected by the unresolved long-period orbit. Some support for a smaller distance to the system is provided by Handler \& Shobbrook (2002), who noted that the Strömgren photometric calibrations yield $M_{v}=0.8 \pm 0.3 \mathrm{mag}$ for this system as a whole. G. Handler (2003, private communication) has computed a value of $M_{v}=1.0$ for component A, making it 3 mag brighter than the canonical value for an F7 dwarf. This result is much more consistent with our spectroscopic results.

From the solar metallicity evolutionary tracks of Schaller et al. (1992), we estimate the primary's mass to be $2.6 M_{\odot}$ for $M_{v}=-0.3$ or $2.0 M_{\odot}$ for $M_{v}=1.0$. Since the significant amplitude of the ellipticity variation indicates that the orbital inclination is not low, we adopt an inclination of $60^{\circ}$. Taking the primary's mass to be in the range $2.0-2.6 M_{\odot}$, combined with the small mass function of only $0.0009 M_{\odot}$ (Table 5), we find a mass of $\sim 0.2 M_{\odot}$ for component $\mathrm{Ab}$, corresponding to a mid-M dwarf. Thus, the total estimated mass of Aa plus $\mathrm{Ab}$ is $\sim 2.5 M_{\odot}$ and that for component $\mathrm{B}$, the $\mathrm{F} 7$ dwarf, is $1.26 M_{\odot}$ (Gray 1992). This results in a mass ratio $\mathrm{B} / \mathrm{A}$ of $\sim 0.5$, in agreement with the preliminary mass ratio of 0.5 determined from the velocity changes of the components in the long period orbit.

We have shown in $\S 3.3$ that the ellipsoidal light variation arises in the A8 component (Aa) of the short-period binary. The higher frequency $\delta$ Scuti variations must arise in the same component since the mid-M secondary $(\mathrm{Ab})$ of the binary is too faint to see and the F7 tertiary component (B) is too cool and, thus, lies well outside the red limit of the $\delta$ Scuti instability strip. The two $\delta$ Scuti periods of 0.06479 and 0.06337 days have a ratio $P_{2} / P_{1}$ of 0.978 , which is inconsistent with typical $\delta$ Scuti period ratios (Breger 1979, Table II) assuming radial pulsation modes. However, as noted in $\S 2.2$, our periods are probably more uncertain than our quoted formal errors since they are only marginally resolved in our analysis. Therefore, our period ratio is subject to considerable uncertainty as well. In any case, the $\delta$ Scuti frequencies are not close to integral multiples of the orbital frequency, suggesting that they are not excited by the tidal distortions of the Aa component raised by the Ab secondary, as may be the case in the eccentric binary HD 177863 (Willems \& Aerts 2002).

XX Pyxidis is another $\delta$ Scuti star that exhibits additional low-frequency variability. Handler et al. (2000) found 22 independent $\delta$ Scuti frequencies in this A4 dwarf from an 81 day multisite photometric campaign; Arentoft, Sterken, \& Handler (2001) found the low-frequency light variation, which they

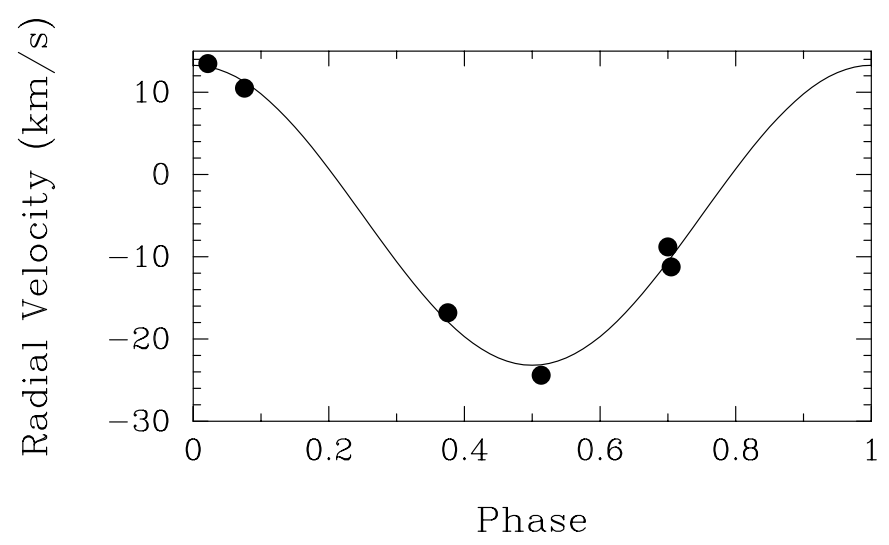

FIG. 6.-Computed radial-velocity curve of HD 207651 Aa compared with the six unit-weight observations. The period is 1.4708 days, twice the ellipticity effect period from photometry. Zero phase is a time of maximum velocity. 
suspected results from duplicity. Aerts et al. (2002) confirmed that the star was a short-period binary, found an orbital period of 1.15 days, and demonstrated that the low-frequency photometric variations were caused by the ellipticity effect. They estimated the unseen binary companion to be an M3 dwarf. An attempt by Pamyatnykh et al. (1998) to model the oscillation modes did not converge on a distinct solution that could satisfactorily fit the observed frequencies. Aerts et al. (2002) surmised this might result from the tidal deformation of the star, causing it to deviate significantly from spherical symmetry assumed in the models.

The low-frequency variation in HD 207651 results from the primary's ellipsoidal shape and not from $\gamma$ Doradus-type pulsation. Thus, like XX Pyx, it is not pulsating simultaneously in $\delta$ Scuti and $\gamma$ Doradus modes. Of the 42 confirmed $\gamma$ Doradus stars (Henry \& Fekel 2003, Table 6), roughly twothirds also occur within the $\delta$ Scuti instability strip (Henry \& Fekel 2003, Figure 28), though the exact fraction depends somewhat on the adopted border of the instability strip and on the photometric system used to plot the stars. At least 22 of the $\gamma$ Doradus stars lying within the $\delta$ Scuti strip have been carefully monitored to search for $\delta$ Scuti variations at the millimagnitude level (Henry et al. 2001; Henry \& Fekel 2002; Handler \& Shobbrook 2002; Fekel \& Henry 2003; Henry \& Fekel 2003). Only one star, HD 209295, exhibits both types of variability, but in that case, the $\gamma$ Doradus pulsations are excited by a companion in a close, elliptical orbit (Handler et al. 2002). Therefore, no star has yet been found that has intrinsic variability of both types.

About $33 \%-50 \%$ of the stars in the $\delta$ Scuti instability strip have $\delta$ Scuti type variability (Breger 2000). This percentage decreases to about $10 \%-15 \%$ near the red edge of the strip where the $\gamma$ Doradus stars overlap (Breger 2000, Fig. 4). However, as Breger (2000) points out, such estimates represent lower limits of $\delta$ Scuti incidence because of the small amplitudes of most $\delta$ Scuti stars and the limited photometric precision of existing surveys. Even with such lower limits, if the two types of variability can exist independently, at least two or three of the 21 carefully monitored $\gamma$ Doradus variables that occur within the $\delta$ Scuti instability strip should exhibit $\delta$ Scuti pulsations. The lack of any such stars strengthens the suggestion of Handler \& Shobbrook (2002) that the two types of pulsation cannot coexist in the same star.

We thank Gerald Handler and Conny Aerts for useful comments on a draft of this paper. We also thank Lou Boyd for his continuing support as Director of Fairborn Observatory and acknowledge the use of the SIMBAD database, operated at CDS, Strasbourg, France. This research is supported by NASA grant NCC5-511 and NSF grant HRD-9706268.
Aerts, C., et al. 2002, MNRAS, 333, L35

Arentoft, T., Sterken, C., \& Handler, G. 2001, MNRAS, 326, 192

Batten, A. H., Fletcher, J. M., \& MacCarthy, D. G. 1989, Publ. Dom. Astrophys. Obs., 17, 1

Breger, M. 1979, PASP, 91, 5

. 2000, in ASP Conf. Ser. 210, Delta Scuti and Related Stars, ed. M. Breger \& M. H. Montgomery (San Francisco: ASP), 3

ESA. 1997, The Hipparcos and Tycho Catalogues (ESA SP-1200) (Noorwijk: ESA)

Fekel, F. C. 1999, in IAU Colloq. 170, Precise Stellar Radial Velocities, ed. J. B. Hearnshaw \& C. D. Scarfe (ASP Conf. Ser. 185; San Francisco: ASP), 378

Fekel, F. C., \& Henry, G. W. 2003, AJ, 125, 2156

Fekel, F. C., Warner, P., \& Kaye, A. B. 2003, AJ, 125, 2196

Fehrenbach, C., Duflot, M., Mannone, C., Burnage, R., \& Genty, V. 1997, A\&AS, 124, 255

Fitzpatrick, M. J. 1993, in ASP Conf. Ser. 52, Astronomical Data Analysis Software and Systems II, ed. R. J. Hanisch, R. V. J. Brissenden, \& J. Barnes (San Francisco: ASP), 472

Gray, D. F. 1992, The Observation and Analysis of Stellar Photospheres (Cambridge: Cambridge Univ. Press)

Handler, G. 1999, MNRAS, 309, L19

Handler, G., et al. 2000, MNRAS, 318, 511

\section{REFERENCES}

Handler, G., et al. 2002, MNRAS, 333, 262

Handler, G., \& Shobbrook, R. R. 2002, MNRAS, 333, 251

Henry, G. W. 1995a, in ASP Conf. Ser. 79, Robotic Telescopes: Current Capabilities, Present Developments, and Future Prospects for Automated Astronomy, ed. G. W. Henry \& J. A. Eaton (San Francisco: ASP), 37 - 1995b, in ASP Conf. Ser. 79, Robotic Telescopes: Current Capabilities, Present Developments, and Future Prospects for Automated Astronomy, ed. G. W. Henry \& J. A. Eaton (San Francisco: ASP), 44

Henry, G. W., \& Fekel, F. C. 2002, PASP, 114, 988

.2003, AJ, 126, 3058

Henry, G. W., Fekel, F. C., Kaye, A. B., \& Kaul, A. 2001, AJ, 122, 3383

Loumos, G. L., \& Deeming, T. J. 1978, Ap\&SS, 56, 285

Matthews, L. D., \& Mathieu, R. D. 1992, in IAU Colloq. 135, Complimentary Approaches to Double and Multiple Star Research, ed. H. A. McAlister \& W. I. Hartkopf (ASP Conf. Ser. 32; San Francisco: ASP), 244

Pamyatnykh, A. A., Dziembowski, W. A., Handler, G., \& Pikall, H. 1998, A\&A, 333, 141

Scarfe, C. D., Batten, A. H., \& Fletcher, J. M. 1990, Publ. Dominion Astrophy. Obs., 18, 21

Schaller, G., Schaerer, D., Meynet, G., \& Maeder, A. 1992, A\&AS, 96, 269

Vanicek, P. 1971, Ap\&SS, 12, 10

Willems, B., \& Aerts, C. 2002, A\&A, 384, 441 